\title{
PERIODICITY IN DIFFUSIVE AGE-STRUCTURED SEIR MODELS
}

\author{
William E. Fitzgibbon, Jeff Morgan, and Mary E. Parrott
}

\begin{abstract}
We consider a diffusive variant of the basic age-structured SEIR epidemic model with time dependent source terms. The source term for susceptibles appears as a forcing term and the source term for the infectives appears as a boundary condition at the age boundary $a=0$. We produce the $a$ priori estimates requisite for guaranteeing the existence of time periodic solutions in response to time periodicity in the forcing functions. Properties of the periodic solutions are also investigated.
\end{abstract}

\section{Introduction}

Our long-range concern is to assess the input of temporal variations on the geographic spread of disease through an age-structured population. Here the concern is to determine how seasonal or daily fluctuations in the ecology of the host or vector populations affect the spread of infection. In the work at hand, we shall confine our attention to a simple diffusive SEIR model and establish the existence of periodic solutions resulting from periodic source terms, removal terms, and contact rates.

Although there has been a considerable amount of work pertaining to periodicity and diffusive systems in mathematical biology, most of it has been concerned with Lotka-Volterra systems, cf. [11] and the references contained therein. Periodicity within the context of population dynamics has been treated in $[12,13,18]$. In [13], periodic solutions are shown for a spatially-dependent Gurtin-MacCamy model with periodic coefficients and a periodic external supply term, and the results are applied to a non-fatal SIR epidemic model. Our work juxtaposes techniques recently developed for diffusive, age-dependent epidemic models $[2,4-6]$ with methods for periodicity in general reaction diffusion systems [14].

The model which we consider will be a variant of the basic age-structured SEIR model where we consider a population subdivided into four subclasses: susceptible, exposed, infective, and removed. We assume that contact between susceptibles and infectives produces exposed individuals and that a "mass action" term proportional to the product of the susceptibles and the infectives governs the transmission from the susceptible to the exposed class. The exposed class consists of individuals in whom the disease is latent and cannot be communicated. The period of latency is assumed to commence immediately upon infection and have duration $\tau>0$. Individuals pass directly from the exposed to the infective state, and they remain infective for the remainder of the duration of the disease. We assume a fixed interval of infectivity $[\tau, \tau+\sigma]$. The removed class consists of individuals who have died or who have

Received June 26, 1997, revised March 23, 1998.

1991 Mathematics Subject Classification: 92D30, 35Q80.

Key words and phrases: periodic solution, SEIR model, age-structure, diffusion. 
acquired permanent immunity by virtue of recovery. In either case, these individuals no longer affect the dynamics of progression of the disease. We assume that individuals are "harvested" to the removed class from both the exposed and the infective classes with a removal rate $\lambda(t)>0$. Individuals who survive the harvesting pass through the age boundary at $\tau+\sigma$. What distinguishes the present situation from a standard age-dependent SEIR epidemic model is the presence of time-periodic sources for the susceptible and infected. The source term for the susceptibles appears as a forcing term on the right-hand side of the equation for susceptibles. The source for the infected has a somewhat more subtle representation because it will portray entry into the process when the age of the infection is zero. We shall assume that our population disperses through a bounded region and remains confined to this region for all time. The mechanism of dispersion is assumed to be random Brownian motion and is approximated in the standard manner by the diffusion operator.

\section{The basic equations and preliminary results}

This section is devoted to preliminary material which places initial-value problems within the framework of abstract evolution equations. The results presented in this section are general in that they require no assumptions on periodicity.

Throughout, $\Omega$ shall be a bounded domain in $R^{n}$ which lies locally on one side of its boundary $\partial \Omega$. We assume that $\partial \Omega$ is an $(n-1)$-dimensional $C^{1+\varepsilon}(0<\varepsilon<1)$ manifold. We introduce three independent variables $x, t$, and $a$ representing the $n$-dimensional spatial position, the elapsed time, and the age of the infection in an individual, respectively. The basic state variables are $u(x, t)$ and $\ell(x, t, a)$ representing the time-dependent spatial density of susceptibles and the time-dependent density of the infection with respect to space and age, respectively. Integration of $\ell(x, t, a)$ with respect to $a$ over the periods of latency and infectivity yields the spatial density of the exposed class $z(x, t)$ and the infective class $v(x, t)$ :

$$
\begin{array}{ll}
z(x, t)=\int_{0}^{\tau} \ell(x, t, a) d a, & x \in \Omega, \quad t \geq 0 \\
v(x, t)=\int_{\tau}^{\tau+\sigma} \ell(x, t, a) d a, & x \in \Omega, \quad t \geq 0
\end{array}
$$

The populations of the susceptible class, $S(t)$, the exposed class, $E(t)$, and the infective class, $I(t)$, can be obtained by integration over $\Omega$ with respect to $x$ :

$$
S(t)=\int_{\Omega} u(x, t) d x, \quad E(t)=\int_{\Omega} z(x, t) d x, \quad I(t)=\int_{\Omega} v(x, t) d x .
$$

The following system of partial differential equations governs the process:

$$
\begin{gathered}
\frac{\partial u(x, t)}{\partial t}=d_{1} \Delta u(x, t)-r(t) u(x, t) v(x, t)+f(x, t) \\
\frac{\partial \ell(x, t, a)}{\partial t}+\frac{\partial \ell(x, t, a)}{\partial a}=d_{2} \Delta \ell(x, t, a)-\lambda(t) \ell(x, t, a)
\end{gathered}
$$

for $x \in \Omega, t>0$, and $a \in[0, \tau+\sigma]$. Homogeneous Neumann boundary conditions,

$$
\frac{\partial u(x, t)}{\partial n}=\frac{\partial \ell(x, t, a)}{\partial n}=0
$$

$x \in \partial \Omega, t>0, a \in(0, \tau+\sigma]$ (where $\partial / \partial n$ denotes the outward normal derivative), insure that the populations remain confined to $\Omega$ for all time. Because the differential 
operator appearing on the left-hand side of $(2.3 \mathrm{~b})$ is first-order hyperbolic, we need to prescribe initial data for $\ell(x, t, a)$ when $t=0$ and $a=0$. We have:

$$
\begin{aligned}
u(x, 0) & =u_{0}(x) & & \text { for } x \in \bar{\Omega}, \\
\ell(x, 0, a) & =\ell_{0}(x, a) & & \text { for } x \in \bar{\Omega}, a \in[0, \tau+\sigma], \\
\ell(x, t, 0):=B(x, t) & =r(t) u(x, t) v(x, t)+h(x, t) & & \text { for } x \in \bar{\Omega}, t>0 .
\end{aligned}
$$

We make the following assumptions on the contact rate, the removal rate, the diffusion coefficients, the initial data, and the terms $f(x, t)$ and $h(x, t)$ :

(i) $r(t), \lambda(t)>0$ for all $t$ and $r(\cdot), \lambda(\cdot) \in C^{1}[0, \infty)$,

(ii) $f(x, t) \geq 0$ for $x \in \Omega, t \geq 0$,

(iii) $h(x, t) \geq 0$ for $x \in \Omega, t \geq 0$, and $h(x, t)>0$ on a set of positive measure in $\Omega \times R^{+}$,

(iv) $f(\cdot, \cdot)$ and $h(\cdot, \cdot)$ are continuously differentiable in each variable,

(v) $u_{0}(x) \geq 0$ for $x \in \bar{\Omega}$, and $u_{0}(\cdot) \in C(\bar{\Omega})$,

(vi) $\ell_{0}(x, a) \geq 0$ for $x \in \bar{\Omega}$ and $a \in[0, \tau+\sigma]$, and $\ell_{0}$ is continuous in the first variable and continuously differentiable in the second variable,

(vii) $d_{1}, d_{2}>0$.

We shall collectively refer to conditions (i)-(vii) as the "given hypotheses."

We say that a pair $(u, \ell)$ is a solution to $(2.3 \mathrm{a})-(2.3 \mathrm{f})$ on $I \subseteq R$ provided $(u(\cdot, t), \ell(\cdot, t, \cdot))$ satisfies $(2.3 \mathrm{a})-(2.3 \mathrm{f})$ for $t \in I$.

We note, however, that because of our techniques of obtaining solutions, namely the method of integrating along characteristics and the method of steps, equation $(2.3 \mathrm{~b})$ will not, in general, hold when $t-a=0, \tau$, or $\tau+\sigma$. If the following compatibility condition is satisfied, then (2.3b) will hold for all $0 \leq a \leq \tau+\sigma$ and $t \geq 0$ :

$$
\ell_{0}(x, 0)=r(0) u_{0}(x) \int_{\tau}^{\tau+\sigma} \ell_{0}(x, a) d a+h(x, 0) .
$$

The following theorem is established by means of arguments similar to those establishing Proposition 2.2 and Remark 2.3 following the proof of this proposition.

Theorem 2.1. If the given hypotheses are satisfied, then for each pair $\left\{u_{0}(x), \ell_{0}\right.$ $(x, a)\}$ there exists a unique, globally-defined solution pair $\{u(x, t), \ell(x, t, a)\}$ of $(2.3 \mathrm{a})$ (2.3f). For each $x \in \bar{\Omega}, t \geq 0, a \in[0, \tau+\sigma], u(x, t) \geq 0$ and $\ell(x, t, a) \geq 0$.

We shall find it convenient to use the abstract theory of linear semigroups and evolution operators. Standard references for this material include [7, 9, 15, 17]. We will work in the Banach space $C(\bar{\Omega})$ and define a time-dependent family of denselydefined closed linear operators, $\{A(t) \mid t \geq 0\}$, by:

$$
(A(t) v)(x)=\left(d_{2} \Delta v-\lambda(t) v\right)(x)
$$

with

$$
D(A(t))=\left\{v \in C(\bar{\Omega}) \mid v \in C^{2}(\bar{\Omega}) \text { and } \frac{\partial v}{\partial n}=0 \text { on } \partial \Omega\right\} .
$$

It is well known that each $A(s)$ so defined is the infinitesimal generator of an analytic semigroup $\left\{T_{s}(t) \mid t \geq 0\right\}$ on $C(\bar{\Omega})$. We let $\left\{T^{0}(t) \mid t \geq 0\right\}$ be the semigroup generated by (2.4a) and (2.4b) when $\lambda(t)=0$. The family $\{A(t) \mid t \geq 0\}$ is the propagator of 
a linear evolution system $\{V(t, s) \mid 0 \leq s \leq t<\infty\}$ on $C(\bar{\Omega})$. This evolution system functions as the solution operator for the time-dependent heat equation associated with (2.4a) and (2.4b), i.e., if $\theta_{0}(\cdot) \in C(\bar{\Omega})$ and $\theta(x, t)=\left(V(t, s) \theta_{0}\right)(x)$, then $\theta(x, t)$ is the classical solution to the linear parabolic initial-boundary value problem,

$$
\begin{aligned}
\frac{\partial \theta(x, t)}{\partial t} & =d_{2} \Delta \theta(x, t)-\lambda(t) \theta(x, t), \quad x \in \Omega, \quad t>s, \\
\frac{\partial \theta}{\partial n} & =0, \quad x \in \partial \Omega, \quad t>s, \\
\theta(x, s) & =\theta_{0}(x), \quad x \in \bar{\Omega} .
\end{aligned}
$$

Elementary arguments give the following representation for $V(t, s)$ :

$$
V(t, s)=\exp \left(-\int_{s}^{t} \lambda(\eta) d \eta\right) T^{0}(t-s) .
$$

Because $\left\{T^{0}(t) \mid t \geq 0\right\}$ is non-expansive, this produces an estimate on the operator norm

$$
\|V(t, s)\|_{\infty, \Omega} \leq \exp \left(-\int_{s}^{t} \lambda(\eta) d \eta\right) \quad \text { for } 0 \leq s \leq t .
$$

We use the evolution system to represent solutions to $(2.3 \mathrm{~b})$.

Proposition 2.2. Let $\{u(x, t), \ell(x, t, a)\}$ be a solution pair of (2.3a)-(2.3f). The solution of $(2.3 \mathrm{~b})$ may be represented along the characteristic line $a-t=c$ in terms of the linear evolution operator $V(\cdot, \cdot)$ as:

$$
\ell(x, t, a)=V(t, 0) \ell_{0}(\cdot, a-t)(x)
$$

for $x \in \Omega, 0<t \leq a \leq \tau+\sigma$, and

$$
\ell(x, t, a)=V(t, t-a)[r(t-a) u(\cdot, t-a) v(\cdot, t-a)+h(\cdot, t-a)](x)
$$

for $x \in \Omega, t>a$, and $a \in[0, \tau+\sigma]$.

Proof. We employ the method of characteristics, cf. $[4,19,20]$. For $c \in R$ and $a-t=c$, we introduce the cohort function,

$$
w_{c}(x, t)=\ell(x, t, t+c) \text {. }
$$

Direct computation yields

$$
\frac{\partial w_{c}(x, t)}{\partial t}=D \ell(x, t, t+c)
$$

where $D=\partial / \partial t+\partial / \partial a$ is the transport derivative. We set $t_{c}=\max \{0,-c\}$ and observe that by $(2.3 \mathrm{~b})$ and $(2.3 \mathrm{c})$, the partial differential equation for the density of infection with respect to age assumes the form

$$
\frac{\partial w_{c}(x, t)}{\partial t}=D \ell(x, t, t+c)=d_{2} \Delta w_{c}(x, t)-\lambda(t) w_{c}(x, t)
$$

for $x \in \Omega, t>t_{c}$, with

$$
\frac{\partial w_{c}}{\partial n}=0, \quad x \in \partial \Omega, \quad t>t_{c}
$$


Solutions to (2.9a) and (2.9b) may be represented by means of the abstract variation of parameters formula,

$$
w_{c}(\cdot, t)=T^{0}\left(t-t_{c}\right) w_{c}\left(\cdot, t_{c}\right)-\int_{t_{c}}^{t} T^{0}(t-s) \lambda(s) w_{c}(\cdot, s) d s
$$

and may also be represented via the evolution operator as

$$
w_{c}(\cdot, t)=V\left(t, t_{c}\right) w_{c}\left(\cdot, t_{c}\right)
$$

If $c=a-t \geq 0$, then $t_{c}=0$, and we have by (2.3e), (2.8), and (2.10),

$$
\begin{aligned}
\ell(\cdot, t, a) & =w_{c}(\cdot, t) \\
& =T^{0}(t) w_{c}(\cdot, 0)-\int_{0}^{t} T^{0}(t-s) \lambda(s) w_{c}(\cdot, s) d s \\
& =T^{0}(t) \ell_{0}(\cdot, a-t)-\int_{0}^{t} T^{0}(t-s) \lambda(s) \ell(\cdot, s, s+a-t) d s .
\end{aligned}
$$

In terms of the evolution operator, we have,

$$
\ell(\cdot, t, a)=V(t, 0) \ell_{0}(\cdot, a-t), \quad t \leq a .
$$

If $c=a-t<0$, then $t_{c}=-c=t-a$, and we have

$$
\begin{aligned}
\ell(\cdot, t, a) & =T_{0}(a) w_{c}(\cdot, t-a)-\int_{t-a}^{t} T^{0}(t-s) \lambda(s) \ell(\cdot, s, s+a-t) d s \\
& =T_{0}(a) \ell(\cdot, t-a, 0)-\int_{t-a}^{t} T^{0}(t-s) \lambda(s) \ell(\cdot, s, s+a-t) d s \\
& =T^{0}(a) B(\cdot, t-a)-\int_{t-a}^{t} T^{0}(t-s) \lambda(s) \ell(\cdot, s, s+a-t) d s
\end{aligned}
$$

Using the evolution operator we have,

$$
\begin{aligned}
\ell(\cdot, t, a) & =V(t, t-a) \ell(\cdot, t-a, 0) \\
& =V(t, t-a) B(\cdot, t-a) \\
& =V(t, t-a)[r(t-a) u(\cdot, t-a) v(\cdot, t-a)+h(\cdot, t-a)], \quad t>a .
\end{aligned}
$$

Remark 2.3. We have shown that if solutions of (2.3a)-(2.3f) exist, then the solution of $(2.3 \mathrm{~b})$ has the representation given by (2.13) and (2.15). Having obtained this representation, it is possible to solve our system (2.3a)-(2.3f) via the method of steps. This procedure is similar to that of the proof of Theorem 3.1 of [4] and so is omitted.

We use the foregoing and (2.1a) and (2.1b) to provide an alternative formulation of $(2.3 \mathrm{a})-(2.3 \mathrm{f})$. If we integrate equation $(2.3 \mathrm{~b})$ with respect to age of the infection over the intervals of latency, $[0, \tau]$, and infectivity, $[\tau, \tau+\sigma]$, we obtain the following 
system:

$$
\begin{aligned}
& \frac{\partial u(x, t)}{\partial t}=d_{1} \Delta u(x, t)-r(t) u(x, t) v(x, t)+f(x, t) \\
& \frac{\partial z(x, t)}{\partial t}=d_{2} \Delta z(x, t)+\ell(x, t, 0)-\ell(x, t, \tau)-\lambda(t) z(x, t) \\
& \quad=d_{2} \Delta z(x, t)+r(t) u(x, t) v(x, t)+h(x, t)-\ell(x, t, \tau)-\lambda(t) z(x, t) \\
& \frac{\partial v(x, t)}{\partial t}=d_{2} \Delta v(x, t)+\ell(x, t, \tau)-\ell(x, t, \tau+\sigma)-\lambda(t) v(x, t)
\end{aligned}
$$

for $x \in \Omega$ and $t>0$, with boundary conditions,

$$
\frac{\partial u(x, t)}{\partial n}=\frac{\partial z(x, t)}{\partial n}=\frac{\partial v(x, t)}{\partial n}=0, \quad x \in \partial \Omega, \quad t>0
$$

and initial conditions,

$$
\begin{aligned}
u(x, 0)=u_{0}(x), \quad z(x, 0) & =z_{0}(x)=\int_{0}^{\tau} \ell_{0}(x, a) d a, \\
v(x, 0) & =v_{0}(x)=\int_{\tau}^{\tau+\sigma} \ell_{0}(x, a) d a, \quad x \in \bar{\Omega} .
\end{aligned}
$$

We note here that the assumption (mentioned in the Introduction) that individuals from both the exposed and infective classes are removed at the same rate, $\lambda(t)$, follows from the assumption of a single underlying infection density function, $\ell$, from which the spatial densities of both classes are defined (cf. (2.1a), (2.1b), and (2.3b)).

We shall find it convenient to let

$$
U(x, t)=(u(x, t), z(x, t), v(x, t))^{T},
$$

and define, for $x \in \Omega, t>\tau+\sigma$,

$$
\begin{aligned}
f_{1}(x, t, U)= & f(x, t)-r(t) u(x, t) v(x, t) \\
f_{2}(x, t, U)= & r(t) u(x, t) v(x, t)-[V(t, t-\tau)(h(\cdot, t-\tau) \\
& \quad+r(t-\tau) u(\cdot, t-\tau) v(\cdot, t-\tau))](x)+h(x, t) \\
f_{3}(x, t, U)=[ & V(t, t-\tau)(h(\cdot, t-\tau)+r(t-\tau) u(\cdot, t-\tau) v(\cdot, t-\tau))](x) \\
& -[V(t, t-\tau-\sigma)(h(\cdot, t-\tau-\sigma) \\
& \quad+r(\cdot, t-\tau-\sigma) u(\cdot, t-\tau-\sigma) v(\cdot, t-\tau-\sigma))](x) .
\end{aligned}
$$

Then we note that by (2.3f) and Proposition 2.2 for $t>\tau+\sigma,(2.16 \mathrm{a})-(2.16 \mathrm{c})$ takes the form

$$
\begin{aligned}
& \frac{\partial u(x, t)}{\partial t}=d_{1} \Delta u(x, t)+f_{1}(x, t, U), \\
& \frac{\partial z(x, t)}{\partial t}=d_{2} \Delta z(x, t)-\lambda(t) z(x, t)+f_{2}(x, t, U), \\
& \frac{\partial v(x, t)}{\partial t}=d_{2} \Delta v(x, t)-\lambda(t) v(x, t)+f_{3}(x, t, U) .
\end{aligned}
$$

Because $A(t)$, as defined by (2.4a) and (2.4b), generates an analytic semigroup, $\left\{T_{t}(s) \mid s \geq 0\right\},-A(t)$ is a sectorial operator and fractional powers of $-A(t)$ may be computed, cf. $[7,9,15,17]$. We set

$$
\widetilde{A}(t)=-A(t)
$$


and for $\alpha>0$, define

$$
\tilde{A}^{-\alpha}(t)=\frac{1}{\Gamma(\alpha)} \int_{0}^{\infty} s^{\alpha-1} T_{t}(s) d s
$$

where $\Gamma(\cdot)$ denotes the Eulerian gamma function. It is known that $\widetilde{A}^{-\alpha}(t)$ is a compact operator on $C(\bar{\Omega})$ for each $t \geq 0$ and has a well-defined, albeit unbounded inverse. We define $\widetilde{A}^{\alpha}(t)=\left(\widetilde{A}^{-\alpha}(t)\right)^{-1}$ and can show that $\widetilde{A}^{\alpha+\beta}(t)=\widetilde{A}^{\alpha}(t) \widetilde{A}^{\beta}(t)$ for $\alpha, \beta \in R$. Moreover, there exists $K(\alpha)>0$ and $\gamma>0$ so that

$$
\left\|\widetilde{A}^{\alpha}(t) V(t, s)\right\|_{\infty, \Omega}<K(\alpha)(t-s)^{-\alpha} e^{-\gamma(t-s)}, \quad \alpha \geq 0, \quad 0 \leq s<t
$$

and

$$
\left\|\widetilde{A}^{\alpha}(r) \widetilde{A}^{-\alpha}(s)\right\|_{\infty, \Omega}<K(\alpha)(r-s), \quad 0<\alpha<1, \quad r, s \geq 0 .
$$

Finally, we know that the operator $V(t, s)$ is a compact mapping of $C(\bar{\Omega})$ to itself, cf. [1]. We have the following compactness result.

Proposition 2.4. Let $\mathcal{A}$ be a bounded subset of $C(\bar{\Omega}) \times C(\bar{\Omega} \times[0, \tau+\sigma])$ and $T_{1}>\tau+\sigma$. If $\mathcal{B}\left(T_{1}\right)$ is the subset of $C(\bar{\Omega}) \times C(\bar{\Omega}) \times C(\bar{\Omega})$ consisting of solutions to $(2.16 \mathrm{a})-(2.16 \mathrm{e})$ at $t=T_{1}$ with initial data $u_{0}(\cdot), z_{0}(\cdot)=\int_{0}^{\tau} \ell_{0}(x, a) d a, v_{0}(\cdot)=\int_{\tau}^{\tau+\sigma} \ell_{0}(x, a) d a$ where $\left\{u_{0}(\cdot), \ell_{0}(\cdot, \cdot)\right\} \in \mathcal{A}$, then $\mathcal{B}\left(T_{1}\right)$ is precompact. Moreover, uniformly bounded trajectories of solutions are precompact.

Proof. We can obtain an a priori estimate for $\left\|u\left(\cdot, T_{1}\right)\right\|_{\infty, \Omega}$ using standard parabolic techniques and use the representation formulae (2.14) and (2.15) to obtain an a priori estimate for $\left\|\ell\left(\cdot, T_{1}, \cdot\right)\right\|_{\infty, \Omega \times[0, \tau+\sigma]}$. This will provide estimates for $\left\|z\left(\cdot, T_{1}\right)\right\|_{\infty, \Omega}$ and $\left\|v\left(\cdot, T_{1}\right)\right\|_{\infty, \Omega}$ by using (2.1a) and (2.1b). In proving that $\mathcal{B}\left(T_{1}\right)$ is precompact, we shall confine our attention to the third component of the equations (2.16a)-(2.16e), namely (2.16c). (The arguments for the other two components are similar.) We have

$$
\begin{aligned}
\frac{\partial v(x, t)}{\partial t} & =d_{2} \Delta v(x, t)-\lambda(t) v(x, t)+\ell(x, t, \tau)-\ell(x, t, \tau+\sigma), \\
v(x, 0) & =v_{0}(x)
\end{aligned}
$$

for $x \in \Omega, 0<t \leq T_{1}$. This may be written abstractly as

$$
\begin{aligned}
\frac{d v(t)}{d t} & =A(t) v(t)+F(\cdot, t), \\
v(0) & =v_{0}
\end{aligned}
$$

where $F(\cdot, t)=\ell(\cdot, t, \tau)-\ell(\cdot, t, \tau+\sigma)$, and where it is understood that the pointwise evaluation $v(t)(x)=v(x, t)$. Solutions to (2.25) have the abstract variation of parameters representation,

$$
v(t)=V(t, 0) v_{0}+\int_{0}^{t} V(t, s) F(\cdot, s) d s
$$

for $0<t \leq T_{1}$. By the boundedness of $\left\{u(\cdot, t), z(\cdot, t), v(\cdot, t) \mid t \in\left[0, T_{1}\right]\right\}$ with initial data $u_{0}(\cdot), z_{0}(\cdot), v_{0}(\cdot) \in \mathcal{A}$ (obtained in a similar fashion as $\mathcal{B}\left(T_{1}\right)$ above), (2.7), and Proposition 2.2, there exists a positive constant $M_{1}$ such that

$$
\sup \left\{\|F(\cdot, t)\|_{\infty, \Omega} \mid t \in\left[0, T_{1}\right]\right\}<M_{1} .
$$


We let $\Pi_{3} \mathcal{B}\left(T_{1}\right)$ be the third component of $\mathcal{B}\left(T_{1}\right)$, and we shall argue that $\widetilde{A}^{\alpha}(0) \Pi_{3}$ $\mathcal{B}\left(T_{1}\right)$ is uniformly bounded for $0<\alpha<1$. If we apply $\widetilde{A}^{\alpha}\left(T_{1}\right)$ to $v\left(T_{1}\right)$ and use (2.26) and (2.22), we produce the estimate,

$$
\begin{aligned}
\left\|\widetilde{A}^{\alpha}\left(T_{1}\right) v\left(T_{1}\right)(\cdot)\right\|_{\infty, \Omega}< & K(\alpha) T_{1}^{-\alpha} e^{-\gamma T_{1}}\left\|v_{0}(\cdot)\right\|_{\infty, \Omega} \\
& +K(\alpha) M_{1} \int_{0}^{T_{1}}\left(T_{1}-s\right)^{-\alpha} e^{-\gamma\left(T_{1}-s\right)} d s \\
< & K(\alpha) T_{1}^{-\alpha} e^{-\gamma T_{1}}\left\|v_{0}(\cdot)\right\|_{\infty, \Omega}+K(\alpha) M_{1} \frac{\Gamma(1-\alpha)}{\gamma^{1-\alpha}} .
\end{aligned}
$$

We set $M_{2}$ equal to the right-hand side of (2.27) and observe that by (2.27) and (2.23),

$$
\begin{aligned}
\left\|\widetilde{A}^{\alpha}(0) \Pi_{3} \mathcal{B}\left(T_{1}\right)\right\|_{\infty, \Omega} & =\left\|\widetilde{A}^{\alpha}(0) \widetilde{A}^{-\alpha}\left(T_{1}\right) \widetilde{A}^{\alpha}\left(T_{1}\right) \Pi_{3} \mathcal{B}\left(T_{1}\right)\right\|_{\infty, \Omega} \\
& <T_{1} K(\alpha) M_{2} .
\end{aligned}
$$

Because $\Pi_{3} \mathcal{B}\left(T_{1}\right)=\widetilde{A}^{-\alpha}(0) \widetilde{A}^{\alpha}(0) \Pi_{3} \mathcal{B}\left(T_{1}\right)$, it is the image of a bounded set under a compact transformation and hence is precompact. The second assertion of the proposition follows from a juxtaposition of the foregoing argument and the proof of Lemma 3.5 in [4].

Proposition 2.5. Let $\{u(x, t), \ell(x, t, a)\}$ be a solution pair to (2.3a)-(2.3f) on $\left[0, T_{1}\right]$ with initial data $\left\{u_{0}(x), \ell_{0}(x, a)\right\}$ for $x \in \Omega$ and $a \in[0, \tau+\sigma]$. If $T_{1}>\tau+\sigma$ and $\mathcal{F}\left(T_{1}\right)$ is the mapping from $C(\bar{\Omega}) \times C(\bar{\Omega} \times[0, \tau+\sigma])$ defined as

$$
\left[\mathcal{F}\left(T_{1}\right)\right]\left(u_{0}(\cdot), \ell_{0}(\cdot, \cdot)\right)(x, a)=\left\{u\left(x, T_{1}\right), \ell\left(x, T_{1}, a\right)\right\},
$$

then $\mathcal{F}\left(T_{1}\right)$ is a compact operator.

Proof. The continuity of the mapping follows from continuous dependence arguments associated with well-posedness. Compactness in the first component is an immediate consequence of the previous proposition and compactness in the second component follows from the representation of $\ell(x, t, a)$ given in Proposition 2.2 and the precompactness of $\mathcal{B}\left(T_{1}\right)$.

The results obtained in this section apply to general systems of the type (2.3a)(2.3f) having time-dependent source terms, mortality rates, and contact rates. For the remainder of the paper, we restrict our attention to the special case where these terms and rates are periodic functions of time.

\section{Periodicity and formulation of the fixed point problem}

We place the following periodicity assumption upon our model:

(P) There exists a $T^{*}>\tau+\sigma$ so that

(i) $\lambda\left(t+T^{*}\right)=\lambda(t), r\left(t+T^{*}\right)=r(t)$ for all $t \geq 0$,

(ii) $f\left(\cdot, t+T^{*}\right)=f(\cdot, t), h\left(\cdot, t+T^{*}\right)=h(\cdot, t)$ for all $t \geq 0$.

The second part of the above stipulates a periodic source term for both the susceptible and the infected populations. From the viewpoint of modeling, we can consider this replenishment due to a fixed periodic spatially-dependent birthrate. We also assume periodicity in the contact and the mortality rates; however, these coefficients may very well be constant. Henceforth, we shall use the symbol $T^{*}$ exclusively to denote the period of the given condition $(\mathrm{P})$. 
By a "periodic solution with period $T^{*}$," we mean a pair $\{u(x, t), \ell(x, t, a)\}$ which satisfies (2.3a)-(2.3f) so that for all $t \geq 0, a \in[0, \tau+\sigma], x \in \Omega$,

(i) $u\left(x, t+T^{*}\right)=u(x, t)$,

(ii) $\ell\left(x, t+T^{*}, a\right)=\ell(x, t, a)$.

Our periodic solutions shall be realized by demonstrating the existence of a fixed point for the mapping $\mathcal{F}\left(T^{*}\right)$. Here, our principle tool will be the following variant of the Schauder Fixed Point Theorem which is given in [8].

Theorem 3.1 (Fixed Point Theorem). Let $X$ be a Banach space and $J: X \rightarrow X$ be a compact map. Assume that there exists a constant $C>0$ such that $\|z\|<C$ for all $z$ satisfying $z=\beta J z$ for $\beta \in(0,1)$. Then there exists a fixed point $z^{*}$ of $J$ such that $\left\|z^{*}\right\| \leq C$.

In the present context, we designate $X=C(\bar{\Omega}) \times C(\bar{\Omega} \times[0, \tau+\sigma])$ with the norm $\|\cdot\|$ computed as the sum of the supremum norms of the components. We set $w_{0}=$ $\left(u_{0}(\cdot), \ell_{0}(\cdot, \cdot)\right)$ and consider solutions to

$$
w_{0}=\beta \mathcal{F}\left(T^{*}\right) w_{0}, \quad 0<\beta<1,
$$

where $\mathcal{F}\left(T^{*}\right)$ is defined by (2.29). The solution set to (3.1) will be denoted by $\Lambda\left(T^{*}\right)$, i.e.,

$$
\Lambda\left(T^{*}\right)=\left\{w_{0} \in X \mid w_{0}=\beta \mathcal{F}\left(T^{*}\right) w_{0} \quad \text { for some } 0<\beta<1\right\} .
$$

Because we showed that $\mathcal{F}\left(T^{*}\right)$ is a compact mapping in Section 2, we can apply the Fixed Point Theorem if we provide a priori estimates for the set $\Lambda\left(T^{*}\right)$. These estimates will be obtained in the subsequent section.

\section{A priori estimates}

In order to obtain a priori estimates for the set $\Lambda\left(T^{*}\right)$, we shall obtain a priori estimates for $u\left(\cdot, T^{*}\right), z\left(\cdot, T^{*}\right)$, and $v\left(\cdot, T^{*}\right)$ using equations $(2.16 \mathrm{a})-(2.16 \mathrm{e})$ and $(2.19 \mathrm{a})-$ (2.19c). Our first result provides $L_{1}$ estimates on the space-time cylinder $\Omega \times\left(0, T^{*}\right)$. In what follows, $\|u(\cdot, \cdot)\|_{1, \Omega \times\left(0, T^{*}\right)}=\int_{0}^{T^{*}} \int_{\Omega} u(x, t) d x d t$.

Lemma 4.1. There exists a constant $C_{1}>0$ so that if $\left(u_{0}(\cdot), \ell_{0}(\cdot, \cdot)\right) \in \Lambda\left(T^{*}\right)$ and

for $x \in \Omega$, then

$$
z_{0}(x)=\int_{0}^{\tau} \ell_{0}(x, a) d a, \quad v_{0}(x)=\int_{\tau}^{\tau+\sigma} \ell_{0}(x, a) d a
$$

$$
\|u(\cdot, \cdot)+z(\cdot, \cdot)+v(\cdot, \cdot)\|_{1, \Omega \times\left(0, T^{*}\right)}<C_{1} .
$$

The constant $C_{1}$ is independent of $u_{0}, \ell_{0}$ and $\beta$.

Proof. We note that if $\left(u_{0}(\cdot), \ell_{0}(\cdot, \cdot)\right) \in \Lambda\left(T^{*}\right)$, then $u_{0}(x)=\beta u\left(x, T^{*}\right)$ and $\ell_{0}(x, a)=$ $\beta \ell\left(x, T^{*}, a\right)$ for some $\beta \in(0,1)$. Thus,

$$
\begin{aligned}
z_{0}(x)=z(x, 0) & =\int_{0}^{\tau} \ell(x, 0, a) d a=\int_{0}^{\tau} \ell_{0}(x, a) d a \\
& =\beta \int_{0}^{\tau} \ell\left(x, T^{*}, a\right) d a=\beta z\left(x, T^{*}\right) .
\end{aligned}
$$


Similarly,

$$
v_{0}(x)=v(x, 0)=\beta v\left(x, T^{*}\right) .
$$

From (2.16a), we have

$$
\frac{\partial u}{\partial t}(x, t)=d_{1} \Delta u(x, t)-r(t) u(x, t) v(x, t)+f(x, t) .
$$

Integrating (4.3) over the space-time cylinder $\Omega \times\left(0, T^{*}\right)$, we obtain

$$
\begin{aligned}
\left\|u\left(\cdot, T^{*}\right)\right\|_{1, \Omega}+\int_{0}^{T^{*}} \int_{\Omega} r(t) u(x, t) v(x, t) d x d t & =\left\|u_{0}(\cdot)\right\|_{1, \Omega}+\|f(\cdot, \cdot)\|_{1, \Omega \times\left(0, T^{*}\right)} \\
& =\beta\left\|u\left(\cdot, T^{*}\right)\right\|_{1, \Omega}+\|f(\cdot, \cdot)\|_{1, \Omega \times\left(0, T^{*}\right)} .
\end{aligned}
$$

This yields

$$
\int_{0}^{T^{*}} \int_{\Omega} r(t) u(x, t) v(x, t) d x d t \leq(\beta-1)\left\|u\left(\cdot, T^{*}\right)\right\|_{1, \Omega}+K<K
$$

where $K=\|f(\cdot, \cdot)\|_{1, \Omega \times\left(0, T^{*}\right)}$. From (2.3b)-(2.3f), we know that the function $\ell(x, t, a)$ satisfies the equations:

$$
\begin{array}{rlrl}
\frac{\partial \ell}{\partial t}+\frac{\partial \ell}{\partial a} & =d_{2} \Delta \ell-\lambda(t) \ell, & & x \in \Omega, \quad t>0, \quad a \in[0, \tau+\sigma], \\
\ell(x, 0, a) & =\ell_{0}(x, a), & & x \in \bar{\Omega}, \quad a \in[0, \tau+\sigma], \\
\ell(x, t, 0) & =r(t) u(x, t) v(x, t)+h(x, t), & & x \in \bar{\Omega}, \quad t>0, \\
\frac{\partial \ell}{\partial n} & =0, & x \in \partial \Omega, \quad t>0, \quad a \in(0, \tau+\sigma] .
\end{array}
$$

We also know that $\ell_{0}(x, a)$ and $r(t) u(x, t) v(x, t)$ are nonnegative for $x \in \bar{\Omega}, t>0$, and $a \in[0, \tau+\sigma]$. We let $L(x, t, a)$ denote the solution to the problem:

$$
\begin{aligned}
\frac{\partial L}{\partial t}+\frac{\partial L}{\partial a} & =d_{2} \Delta L-\lambda(t) L, & & x \in \Omega, \quad t>0, \quad a \in[0, \tau+\sigma], \\
L(x, 0, a) & =0, & & x \in \bar{\Omega}, \quad a \in[0, \tau+\sigma], \\
L(x, t, 0) & =h(x, t), & & x \in \bar{\Omega}, \quad t>0, \\
\frac{\partial L}{\partial n} & =0, & & x \in \partial \Omega, \quad t>0, \quad a \in(0, \tau+\sigma] .
\end{aligned}
$$

( $L$ is obtained in a similar manner as $\ell$.) By the comparison principle, $\ell(x, t, a) \geq$ $L(x, t, a)$. Furthermore, since we have $h(x, t)$ nonnegative and not identically zero on $\Omega \times\left(0, T^{*}\right)$, we must have $L(x, t, a)$ positive on some open subset of $\Omega \times\left(0, T^{*}\right) \times$ $(\tau, \tau+\sigma)$. As a result,

$$
v(x, t)=\int_{\tau}^{\tau+\sigma} \ell(x, t, a) d a \geq \int_{\tau}^{\tau+\sigma} L(x, t, a) d a .
$$

This yields the fact that $v(x, t)$ is positive and bounded below on some open subset of $\Omega \times\left(0, T^{*}\right)$. Since $r$ is positive, we can find an open subset of $\Omega \times\left(0, T^{*}\right)$, which we call $E$, and a positive constant $\delta$ such that

$$
r(t) v(x, t)>\delta \text { for }(x, t) \in E .
$$


We now multiply equation (4.3) by $u$ and integrate over $\Omega \times\left(0, T^{*}\right)$ to obtain

$$
\begin{gathered}
u \frac{\partial u}{\partial t}-d_{1} u \Delta u+r u^{2} v=u f, \\
\left\|u^{2}\left(\cdot, T^{*}\right)\right\|_{1, \Omega}+2 d_{1} \int_{0}^{T^{*}} \int_{\Omega}|\nabla u|^{2} d x d t+2 \int_{0}^{T^{*}} \int_{\Omega} r u^{2} v d x d t \\
=\left\|u_{0}^{2}(\cdot)\right\|_{1, \Omega}+2 \int_{0}^{T^{*}} \int_{\Omega} u f d x d t .
\end{gathered}
$$

Using the fact that $u_{0}(\cdot)=\beta u\left(\cdot, T^{*}\right)$ for some $\beta \in(0,1),(4.5)$, and Young's inequality, (4.6) yields

$$
\begin{aligned}
2 d_{1} \int_{0}^{T^{*}} \int_{\Omega}|\nabla u|^{2} d x d t+2 \delta \int_{0}^{T^{*}} \int_{E} u^{2}(x, t) d x d t & <2 \int_{0}^{T^{*}} \int_{\Omega} u f d x d t \\
& <\varepsilon \int_{0}^{T^{*}} \int_{\Omega} u^{2} d x d t+\frac{1}{\varepsilon} \int_{0}^{T^{*}} \int_{\Omega} f^{2} d x d t
\end{aligned}
$$

for $\varepsilon>0$. We know that (cf., for example, [16, Chapter 11A]) $u(x, t)=\bar{u}(t)+u^{*}(x, t)$ where $\bar{u}(t)=\frac{1}{|\Omega|} \int_{\Omega} u(x, t) d x$, and the residue $u^{*}(x, t)$ has the property that

$$
\int_{\Omega}\left(u^{*}(x, t)\right)^{2} d x \leq a_{1} \int_{\Omega}|\nabla u(x, t)|^{2} d x
$$

for a positive constant $a_{1}$. Using Young's inequality, we have

$$
\int_{\Omega} u^{2}(x, t) d x \leq a_{2} \int_{\Omega} \bar{u}^{2}(t) d x+a_{3} \int_{\Omega}\left(u^{*}(x, t)\right)^{2} d x
$$

for some positive constants $a_{2}$ and $a_{3}$. Since

$$
\int_{\Omega} \bar{u}^{2}(t) d x=|\Omega| \bar{u}^{2}(t)=\frac{|\Omega|}{|E|} \int_{E} \bar{u}^{2}(t) d x
$$

and

$$
\int_{E} \bar{u}^{2}(t) d x \leq a_{4} \int_{E} u^{2}(x, t) d x+a_{5} \int_{E}\left(u^{*}(x, t)\right)^{2} d x
$$

for some positive constants $a_{4}$ and $a_{5},(4.8)-(4.11)$ yield

$$
\int_{\Omega} u^{2}(x, t) d x \leq K_{1} \int_{E} u^{2}(x, t) d x+K_{2} \int_{\Omega}|\nabla u(x, t)|^{2} d x
$$

for some positive constants $K_{1}$ and $K_{2}$. Consequently, if we choose $\varepsilon$ sufficiently small in (4.7), then we obtain the bounds

$$
\int_{0}^{T^{*}} \int_{\Omega} u^{2}(x, t) d x d t \leq K_{3}
$$

and

$$
\int_{0}^{T^{*}} \int_{\Omega}|\nabla u(x, t)|^{2} d x d t \leq K_{4}
$$


for some positive constants $K_{3}$ and $K_{4}$. By the Cauchy-Schwarz inequality, (4.13) yields a positive constant $K_{5}$ such that

$$
\int_{0}^{T^{*}} \int_{\Omega} u(x, t) d x d t \leq K_{5} .
$$

We integrate equation (2.16b) over $\Omega \times\left(0, T^{*}\right)$ to obtain

$$
\begin{aligned}
& \left\|z\left(\cdot, T^{*}\right)\right\|_{1, \Omega}+\int_{0}^{T^{*}} \int_{\Omega} \ell(x, t, \tau) d x d \tau+\int_{0}^{T^{*}} \int_{\Omega} \lambda(t) z(x, t) d x d t \\
& \leq\left\|z_{0}(\cdot)\right\|_{1, \Omega}+\int_{0}^{T^{*}} \int_{\Omega} r(t) u(x, t) v(x, t) d x d t
\end{aligned}
$$

which yields, by (4.1) and (4.4),

$$
\int_{0}^{T^{*}} \int_{\Omega} \lambda(t) z(x, t) d x d t \leq K
$$

and

$$
\int_{0}^{T^{*}} \int_{\Omega} \ell(x, t, \tau) d x d t \leq K
$$

Since $\lambda$ is positive and bounded on $\left[0, T^{*}\right],(4.17)$ yields

$$
\int_{0}^{T^{*}} \int_{\Omega} z(x, t) d x d t \leq K_{6}
$$

for a positive constant $K_{6}$. In a similar fashion, we obtain, upon integrating equation (2.16c) and using (4.2) and (4.18),

$$
\int_{0}^{T^{*}} \int_{\Omega} v(x, t) d x d t \leq K_{7}
$$

for a positive constant $K_{7}$. The bounds (4.15), (4.19), and (4.20) yield the conclusion of the lemma.

Lemma 4.2. There exists a constant $C_{2}>0$ so that if $\left(u_{0}(\cdot), \ell_{0}(\cdot, \cdot)\right) \in \Lambda\left(T^{*}\right)$, then

$$
\max \left\{\left\|u\left(\cdot, T^{*}\right)\right\|_{\infty, \Omega},\left\|\ell\left(\cdot, T^{*}, \cdot\right)\right\|_{\infty, \Omega \times[0, \tau+\sigma]}\right\}<C_{2}
$$

The constant $C_{2}$ is independent of $u_{0}, \ell_{0}$, and $\beta$.

Proof. We begin working with equation $(2.16 \mathrm{a})$. We let $y_{1}(\cdot, \cdot)$ be the Green's function associated with the problem

$$
\begin{aligned}
& \frac{\partial \rho}{\partial t}(x, t)=d_{1} \Delta \rho(x, t), \quad x \in \Omega, \quad t>0, \\
& \frac{\partial \rho}{\partial n}(x, t)=0, \quad x \in \partial \Omega, \quad t>0 .
\end{aligned}
$$

Using the Duhamel formula, we have

$$
\begin{gathered}
u\left(x, T^{*}\right)=\int_{\Omega} g_{1}\left(x-y, T^{*}\right) u_{0}(y) d y+\int_{0}^{T^{*}} \int_{\Omega} g_{1}\left(x-y, T^{*}-s\right) f(y, s) d y d s \\
-\int_{0}^{T^{*}} \int_{\Omega} g_{1}\left(x-y, T^{*}-s\right) r(s) u(y, s) v(y, s) d y d s
\end{gathered}
$$


Hence,

$$
\left\|u\left(\cdot, T^{*}\right)\right\|_{\infty, \Omega} \leq\left\|g_{1}\left(\cdot, T^{*}\right)\right\|_{\infty, \Omega}\left\|u_{0}(\cdot)\right\|_{1, \Omega}+\|f(\cdot, \cdot)\|_{\infty, \Omega \times\left(0, T^{*}\right)} .
$$

Here we have used the positivity of $g, r, u, v$ and the fact that

$$
\left\|\int_{\Omega} g_{1}\left(\cdot-y, T^{*}-s\right) f(y, s) d y\right\|_{\infty, \Omega} \leq\|f(\cdot, s)\|_{\infty, \Omega} .
$$

We now need an a priori bound on $\left\|u_{0}(\cdot)\right\|_{1, \Omega}$ for $\left(u_{0}(\cdot), \ell_{0}(\cdot, \cdot)\right) \in \Lambda\left(T^{*}\right)$. The Mean Value Theorem, together with Lemma 4.1, guarantees that there exists an $\eta^{*} \in\left[0, T^{*}\right]$ so that

$$
\left\|u\left(\cdot, \eta^{*}\right)\right\|_{1, \Omega} \leq \frac{C}{T^{*}}
$$

for a positive constant $C$. Then, similar to (4.22), we have

$$
\begin{gathered}
u\left(x, T^{*}\right)=\int_{\Omega} g_{1}\left(x-y, T^{*}-\eta^{*}\right) u\left(y, \eta^{*}\right) d y+\int_{\eta^{*}}^{T^{*}} \int_{\Omega} g_{1}\left(x-y, T^{*}-s\right) f(y, s) d y d s \\
-\int_{\eta^{*}}^{T^{*}} \int_{\Omega} g_{1}\left(x-y, T^{*}-s\right) r(s) u(y, s) v(y, s) d y d s .
\end{gathered}
$$

Using (4.25), this yields

$$
\left\|u\left(\cdot, T^{*}\right)\right\|_{1, \Omega} \leq\left\|u\left(\cdot, \eta^{*}\right)\right\|_{1, \Omega}+\int_{\eta^{*}}^{T^{*}} \int_{\Omega} f(y, s) d y d s=\widetilde{C}
$$

for a positive constant $\widetilde{C}$. Here we have used the fact that

$$
\left\|\int_{\Omega} g_{1}\left(\cdot-y, T^{*}-\eta^{*}\right) u\left(y, \eta^{*}\right) d y\right\|_{1, \Omega} \leq\left\|u\left(\cdot, \eta^{*}\right)\right\|_{1, \Omega} .
$$

Since $\left(u_{0}(\cdot), \ell_{0}(\cdot, \cdot)\right) \in \Lambda\left(T^{*}\right), u_{0}(\cdot)=\beta u\left(\cdot, T^{*}\right)$ for some $0<\beta<1$. Thus, from (4.27), we have

$$
\left\|u_{0}(\cdot)\right\|_{1, \Omega} \leq \widetilde{C}
$$

The $L_{\infty}$ bound for $u\left(\cdot, T^{*}\right)$ now follows from (4.23) and (4.29).

We now wish to produce an a priori bound for $\left\|\ell\left(\cdot, T^{*}, \cdot\right)\right\|_{\infty, \Omega \times[0, \tau+\sigma]}$. To this end, we begin by producing an a priori bound for $w(x, t)=z(x, t)+v(x, t)$. Again, using the Mean Value Theorem and Lemma 4.1, we see that there exists an $\eta^{*} \in\left[0, T^{*}\right]$ and a positive constant $K$ so that

$$
\left\|w\left(\cdot, \eta^{*}\right)\right\|_{1, \Omega} \leq \frac{K}{T^{*}}
$$

We return to equation (2.16a) and integrate on $\Omega \times(0, t), t \in\left(0, T^{*}\right]$, to obtain

$$
\|u(\cdot, t)\|_{1, \Omega}+\int_{0}^{t} \int_{\Omega} r(s) u(x, s) v(x, s) d x d s=\left\|u_{0}(\cdot)\right\|_{1, \Omega}+\int_{0}^{t} \int_{\Omega} f(x, s) d x d s .
$$

The bound obtained in (4.29) for $\left\|u_{0}(\cdot)\right\|_{1, \Omega}$ will yield a positive constant $K_{1}$ so that

$$
\sup _{t \in\left[0, T^{*}\right]} \int_{0}^{t} \int_{\Omega} r(s) u(x, s) v(x, s) d x d s \leq K_{1} \text {. }
$$


By adding equations (2.16b) and (2.16c), we see that $w=z+v$ satisfies

$$
\frac{\partial w}{\partial t}(x, t)=d_{2} \Delta w(x, t)+\ell(x, t, 0)-\lambda(t) w(x, t)-\ell(x, t, \tau+\sigma) .
$$

Using the positivity of $\ell(\cdot, \cdot, \cdot)$ and $w(\cdot, \cdot)$, and (2.3f), we integrate (4.33) over $\Omega \times\left(\eta^{*}, t\right), t \in\left[\eta^{*}, T^{*}\right]$, to observe that

$$
\|w(\cdot, t)\|_{1, \Omega} \leq\left\|w\left(\cdot, \eta^{*}\right)\right\|_{1, \Omega}+\int_{\eta^{*}}^{t} \int_{\Omega} h(x, s) d x d s+\int_{\eta^{*}}^{t} \int_{\Omega} r(s) u(x, s) v(x, s) d x d s .
$$

Consequently, by (4.30) and (4.32), we are assured a positive constant $K_{2}$ so that

$$
\left\|w\left(\cdot, T^{*}\right)\right\|_{1, \Omega} \leq K_{2}
$$

and because $\left(u_{0}(\cdot), \ell_{0}(\cdot, \cdot)\right) \in \Lambda\left(T^{*}\right)$, we can conclude, upon using (4.1) and (4.2), that

$$
\left\|w_{0}(\cdot)\right\|_{1, \Omega} \leq K_{2}
$$

By using the Duhammel formula and (4.3), similar to (4.22), we find for $t \in\left(0, T^{*}\right]$,

$$
\|u(\cdot, t)\|_{\infty, \Omega} \leq\left\|u_{0}(\cdot)\right\|_{\infty, \Omega}+\|f(\cdot, \cdot)\|_{\infty, \Omega \times\left(0, T^{*}\right)} .
$$

Thus, there is a positive constant $K_{3}$ so that

$$
\|u(\cdot, t)\|_{\infty, \Omega} \leq K_{3}
$$

for $t \in\left[0, T^{*}\right]$ where we have again used the fact that $\left(u_{0}(\cdot), \ell_{0}(\cdot, \cdot)\right) \in \Lambda\left(T^{*}\right)$, which yields a uniform bound on $u_{0}(\cdot)$ from (4.23) and (4.29). Because of (4.37) and the fact that $0 \leq v(x, t) \leq w(x, t)=z(x, t)+v(x, t)$, we obtain from (4.33),

$$
\begin{aligned}
\frac{\partial w}{\partial t}(x, t) & \leq d_{2} \Delta w(x, t)+K_{4} w(x, t)+h(x, t), \quad x \in \Omega, \quad t \in\left(0, T^{*}\right], \\
\frac{\partial w}{\partial n}(x, t) & =0, \quad x \in \partial \Omega, \quad t \in\left(0, T^{*}\right], \\
w(x, 0) & =w_{0}(x)=z_{0}(x)+v_{0}(x), \quad x \in \bar{\Omega},
\end{aligned}
$$

where $K_{4}=K_{3} \sup _{t \in\left[0, T^{*}\right]} r(t)$. We let $g_{2}(\cdot, \cdot)$ be the Green's function associated with the problem

$$
\begin{aligned}
& \frac{\partial \rho}{\partial t}(x, t)=d_{2} \Delta \rho(x, t)+K_{4} \rho(x, t), \quad x \in \Omega, \quad t \in\left(0, T^{*}\right] \\
& \frac{\partial \rho}{\partial n}(x, t)=0, \quad x \in \partial \Omega, \quad t \in\left(0, T^{*}\right] .
\end{aligned}
$$

If we let $\theta(x, t)$ satisfy the equation

$$
\theta(x, t)=\int_{\Omega} g_{2}(x-y, t) w_{0}(y) d y+\int_{0}^{t} \int_{\Omega} g_{2}(x-y, t-s) h(y, s) d y d s,
$$

then the comparison principle will yield

$$
0 \leq w(x, t) \leq \theta(x, t) .
$$

Hence, we may apply the Hölder inequality to observe that

$$
\left\|w\left(\cdot, T^{*}\right)\right\|_{\infty, \Omega} \leq\left\|g_{2}\left(\cdot, T^{*}\right)\right\|_{\infty, \Omega}\left\|w_{0}(\cdot)\right\|_{1, \Omega}+\|h(\cdot, \cdot)\|_{\infty, \Omega \times\left(0, T^{*}\right)} .
$$


Using (4.36), the above yields a positive constant $K_{5}$ so that

$$
\left\|w\left(\cdot, T^{*}\right)\right\|_{\infty, \Omega} \leq K_{5}
$$

and also

$$
\left\|w_{0}(\cdot)\right\|_{\infty, \Omega} \leq K_{5}
$$

Returning to (4.40) and (4.41), we observe that

$$
\|w(\cdot, t)\|_{\infty, \Omega} \leq\left\|w_{0}(\cdot)\right\|_{\infty, \Omega}+\|h(\cdot, \cdot)\|_{\infty, \Omega \times\left(0, T^{*}\right)}
$$

for $t \in\left[0, T^{*}\right]$. Thus, we have the uniform bounds on $z(\cdot, t)$ and $v(\cdot, t)$ given by

$$
\|z(\cdot, t)\|_{\infty, \Omega} \leq K_{6}
$$

and

$$
\|v(\cdot, t)\|_{\infty, \Omega} \leq K_{6}
$$

for $t \in\left[0, T^{*}\right]$ where $K_{6}=K_{5}+\|h(\cdot, \cdot)\|_{\infty, \Omega \times\left(0, T^{*}\right)}$. We recall from Proposition 2.2 that if $a \in[0, \tau+\sigma]$, then

$$
\begin{aligned}
\ell\left(x, T^{*}, a\right)=[ & V\left(T^{*}, T^{*}-a\right)\left(h\left(\cdot, T^{*}-a\right)\right. \\
& \left.\left.+r\left(T^{*}-a\right) u\left(\cdot, T^{*}-a\right) v\left(\cdot, T^{*}-a\right)\right)\right](x) .
\end{aligned}
$$

By virtue of the bounds on $\left\|u\left(\cdot, T^{*}\right)\right\|_{\infty, \Omega}$ and $\left\|v\left(\cdot, T^{*}\right)\right\|_{\infty, \Omega}$ (from (4.23) and (4.47)), we have $K_{7}>0$ so that

$$
\left\|\ell\left(\cdot, T^{*}, \cdot\right)\right\|_{\infty, \Omega \times[0, \tau+\sigma]} \leq K_{7} .
$$

This yields, via the fact that $\left(u_{0}(\cdot), \ell_{0}(\cdot, \cdot)\right) \in \Lambda\left(T^{*}\right)$, the uniform bound

$$
\left\|\ell_{0}(\cdot, \cdot)\right\|_{\infty, \Omega \times[0, \tau+\sigma]} \leq K_{7}
$$

Recent work, $[2,4-6]$, has produced the somewhat surprising observation that in many instances, it is much simpler to obtain a priori estimates for systems which involve both age dependence and diffusion than it is for systems which only involve diffusion. The case at hand illustrates this point. System (2.16a)-(2.16e) may be viewed as a system of reaction-diffusion type involving delays. The recent paper [14] establishes criteria sufficient to guarantee the existence of periodic solutions to reaction diffusion systems. It would have been possible to adapt the techniques from [14] and to use duality arguments coupled with an iteration producing $L_{p}$ space-time cylinder bounds for an increasing sequence of $p$ 's to obtain the desired a priori $L_{\infty}$ bound for $\ell\left(x, T^{*}, a\right)$. However, the argument given here is much simpler and more straightforward. We are led to conjecture that estimates obtained for reaction-diffusion systems with an "artificial age dependence" may be used to approximate estimates for reaction-diffusion systems in a manner analogous to the estimates obtained via approximation of conservation laws by vanishing viscosity arguments. 


\section{Main results}

We now provide sufficient conditions to yield periodic solutions.

Theorem 5.1. Assume the given hypothesis (i)-(vii) of Section 2 and (P) of Section 3. Then there exists a solution pair $\{u(x, t), \ell(x, t, a)\}$ to (2.3a)-(2.3f) so that for $t \geq 0, a \in[0, \tau+\sigma], x \in \Omega$, we have

$$
u\left(x, t+T^{*}\right)=u(x, t), \quad \ell\left(x, t+T^{*}, a\right)=\ell(x, t, a) .
$$

Proof. We have demonstrated that the set $\left\{\mathcal{F}\left(T^{*}\right) w_{0} \mid w_{0} \in \Lambda\left(T^{*}\right)\right\}$ where $\mathcal{F}\left(T^{*}\right)$ is defined by (2.29) is a bounded subset of the Banach space $X=C(\bar{\Omega}) \times C(\bar{\Omega} \times[0, \tau+\sigma])$ and that the mapping $\mathcal{F}\left(T^{*}\right)$ is compact on $X$. Thus the hypotheses of the Fixed Point Theorem stated in Section 3 hold. Therefore, there exists $\left(u_{0}(\cdot), \ell_{0}(\cdot, \cdot)\right) \in X$ and an associated solution pair $(u(x, t), \ell(x, t, a))$ so that for $x \in \Omega$ and $a \in[0, \tau+\sigma]$,

$$
u\left(x, T^{*}\right)=u_{0}(x), \quad \ell\left(x, T^{*}, a\right)=\ell_{0}(x, a) .
$$

If we let $\left(u^{*}(x, t), \ell^{*}(x, t, a)\right)=\left(u\left(x, t+T^{*}\right), \ell\left(x, t+T^{*}, a\right)\right)$, we can apply the uniqueness result of Theorem 2.1 to insure that $(u(x, t), \ell(x, t, a))=\left(u\left(x, t+T^{*}\right)\right.$, $\left.\ell\left(x, t+T^{*}, a\right)\right)$, and we directly infer the existence of a periodic solution.

It is evident that $z\left(x, t+T^{*}\right)=z(x, t)$ and $v\left(x, t+T^{*}\right)=v(x, t)$, and because $T^{*}>0$, we have by $(2.3 \mathrm{f})$

$$
\begin{aligned}
\left(u\left(x, T^{*}\right), \ell\left(x, T^{*}, 0\right)\right) & =\left(u_{0}(x), \ell_{0}(x)\right) \\
& =\left(u\left(x, T^{*}\right), r\left(T^{*}\right) u\left(x, T^{*}\right) v\left(x, T^{*}\right)+h\left(x, T^{*}\right)\right) \\
& =(u(x, 0), r(0) u(x, 0) v(x, 0)+h(x, 0)) .
\end{aligned}
$$

Hence, we see that the compatibility condition (C) holds. We obtain further regularity results by applying standard parabolic regularity theory to $(2.19 \mathrm{~b})$ and $(2.19 \mathrm{c})$. It can be readily shown that $v(\cdot, t)$ is eventually $C^{1}$, and we can use periodicity and condition (C) to infer that $\ell(\cdot, t, a)$ is $C^{1}$ for $a \in[0, \tau+\sigma]$.

\section{Further considerations}

We have established the existence of a periodic solution to our system (2.3a)-(2.3f) under the condition that the periodic source terms $f(x, t)$ and $h(x, t)$ are nonnegative for $x \in \Omega, t \in\left[0, T^{*}\right]$, and $h(x, t)>0$ on a set of positive measure in $\Omega \times\left[0, T^{*}\right]$. We now look at the cases where one or both of these source terms is zero, and try to obtain some qualitative feel for the nature of the periodic solutions. We shall refer to a "trivial periodic solution" as one having the form:

$$
u(x, t)=\text { constant, } \quad \ell(x, t, a)=0
$$

for $x \in \Omega, t \geq 0$, and $a \in[0, \tau+\sigma]$. We have the following result:

Proposition 6.1. Assume that the hypotheses (i), (iv)-(vii), (P) of Sections 2 and 3 hold.

(a) If $f(x, t)=h(x, t)=0$ for all $x \in \Omega$ and $t \in\left[0, T^{*}\right]$, then every periodic solution is a trivial periodic solution, and every solution to the initial-value problem (2.3a)-(2.3f) converges to a trivial periodic solution. If $u_{0}(x)>0$ on a set of positive measure, then the constant of (6.1) is positive. 
(b) If $f(x, t)=0$ and $h(x, t)>0$ for $x \in \Omega$ and $t \in\left[0, T^{*}\right]$, then every uniformly bounded solution to (2.3a)-(2.3f) is attracted to the periodic trajectory

$$
\theta^{*}(x, a)=\left\{\left(0,\left[V\left(T^{*}+s, T^{*}+s-a\right) h\left(\cdot, T^{*}+s-a\right)\right](x)\right) \mid s \in\left[0, T^{*}\right]\right\} .
$$

(c) If $f(x, t)>0$ for $(x, t) \in a$ set of positive measure in $\Omega \times\left[0, T^{*}\right]$, and if $(u(x, t), \ell(x, t, a))$ is a periodic solution pair, then

$$
v(x, t)=\int_{\tau}^{\tau+\sigma} \ell(x, t, a) d a \neq 0 .
$$

Proof. Assertion (c) is the easiest to prove. Assume that $(u(x, t), 0)$ is a periodic solution. Then $v(x, t)=0$ and (2.3a) becomes

$$
\frac{\partial u(x, t)}{\partial t}=d_{1} \Delta u(x, t)+f(x, t) .
$$

Integrating over $\Omega \times\left(0, T^{*}\right)$, we find that

$$
0=\left\|u\left(\cdot, T^{*}\right)\right\|_{1, \Omega}-\left\|u_{0}(\cdot)\right\|_{1, \Omega}=\int_{0}^{T^{*}}\|f(\cdot, t)\|_{1, \Omega} d t,
$$

which is a contradiction. Thus, if $f(x, t)>0$ on a set of positive measure and $(u(x, t), \ell(x, t, a))$ is a periodic solution, then $\ell(x, t, a) \neq 0$ and $v(x, t) \neq 0$.

To prove (a), we let $U(x, t)$ denote the solution triple to $(2.16 \mathrm{a})-(2.16 \mathrm{e})$ where (2.16a) $-(2.16 \mathrm{c})$ have the form $(2.19 \mathrm{a})-(2.16 \mathrm{c})$ for $t>\tau+\sigma$. We can adapt the argument of Theorem 3.3 of [4] to observe that, if $u_{0}(x)>0$ on a set of positive measure, then there exists a positive constant $u^{*}$ so that $\lim _{t \rightarrow \infty} U(x, t)=\left(u^{*}, 0,0\right)$ and $\lim _{t \rightarrow \infty} \ell(x, t, a)=0$ uniformly in $x \in \Omega, a \in[0, \tau+\sigma]$. Thus, there are no nontrivial periodic solutions, and every solution of $(2.3 \mathrm{a})-(2.3 \mathrm{e})$ converges to a trivial periodic solution.

To establish (b), we begin by observing that equation (2.3a) becomes

$$
\frac{\partial u(x, t)}{\partial t}=d_{1} \Delta u(x, t)-r(t) u(x, t) v(x, t)
$$

with homogeneous Neumann boundary conditions. Under the assumption of uniform bounds on $u(x, t)$ and $\ell(x, t, a)$ (and consequently on $v(x, t)$ ), we can adopt the invariance methods used in Chapter 14 of [16] to argue that $|\nabla u(x, t)|$ and $\left|\nabla u_{t}(x, t)\right|$ are uniformly bounded, and then apply the arguments in [3] to infer that $u(x, t)$ converges uniformly to its spatial average $\bar{u}(t)$; i.e., if

$$
\bar{u}(t)=\frac{1}{|\Omega|} \int_{\Omega} u(x, t) d x
$$

then

$$
\lim _{t \rightarrow \infty}\|u(x, t)-\bar{u}(t)\|_{\infty, \Omega}=0 .
$$

Integration of (6.2) over $\Omega$ immediately yields that $\bar{u}(t)$ is nonincreasing in $t$ and hence must converge to a limit $u^{*} \geq 0$, and therefore,

$$
\lim _{t \rightarrow \infty}\left\|u(\dot{x}, t)-u^{*}\right\|_{\infty, \Omega}=0 .
$$


We may use the representation given by Proposition 2.2 along with the fact that $h(x, t)$ is strictly positive and periodic to observe that there exists an $\varepsilon>0$ such that

$$
\ell(x, t, a)>\varepsilon
$$

whenever $t>\tau+\sigma, a \in[0, \tau+\sigma]$, and $x \in \Omega$. The integration of (6.2) over $\Omega \times(0, t)$ yields

$$
\|u(\cdot, t)\|_{1, \Omega}+\int_{0}^{t} \int_{\Omega} r(s) u(x, s) v(x, s) d x d s=\left\|u_{0}(\cdot)\right\|_{1, \Omega} .
$$

This implies that

$$
\int_{0}^{t} \int_{\Omega} r(s) u(x, s) v(x, s) d x d s=\int_{0}^{t} \int_{\Omega} r(s)\left(\int_{\tau}^{\tau+\sigma} \ell(x, s, a) d a\right) u(x, s) d x d s<\infty .
$$

Letting $r^{*}=\inf \left\{r(t) \mid t \in\left[0, T^{*}\right]\right\}$, and using (6.5), we see that

$$
\int_{0}^{t} \int_{\Omega} r(s)\left(\int_{\tau}^{\tau+\sigma} \ell(x, s, a) d a\right) u(x, s) d x d s>r^{*} \varepsilon \sigma \int_{0}^{t}\|u(\cdot, s)\|_{1, \Omega} d s,
$$

which implies by (6.6) that

$$
\int_{0}^{t}\|u(\cdot, s)\|_{1, \Omega} d s<\frac{1}{r^{*} \varepsilon \sigma} \int_{0}^{t} \int_{\Omega} r(s)\left(\int_{\tau}^{\tau+\sigma} \ell(x, s, a) d a\right) u(x, s) d x d s<\infty .
$$

This further implies that $\limsup _{t \rightarrow \infty}\|u(\cdot, t)\|_{1, \Omega}=0$. However, $u(\cdot, t)$ converges to the constant function $u^{*}$ in $C(\bar{\Omega})$ and thus must converge to $u^{*}$ in $L_{1}(\Omega)$, and we may thereby conclude that $u^{*}=0$ and that $u(\cdot, t)$ converges uniformly to zero.

We combine the arguments of Proposition 2.4 and Lemma 3.5 of [4] to argue the precompactness of the set $\{v(\cdot, t) \mid t \geq 0\}$ in $C(\bar{\Omega})$. Because we have seen that $u(\cdot, t)$ converges, our representation formula from Proposition 2.2 shows that $\{\ell(\cdot, t, \cdot) \mid t \geq$ $0\}$ is precompact in $C(\bar{\Omega} \times[0, \tau+\sigma])$, and we have thereby shown that the trajectory $\theta=\{(u(\cdot, t), \ell(\cdot, t, \cdot)) \mid t \geq 0\}$ is precompact in $X$. Letting $w_{0}=\left(u_{0}(\cdot), \ell_{0}(\cdot, \cdot)\right)$, we may draw upon well-known results in dynamical systems theory, cf. [10], to see that $\theta$ has a nonempty, compact, connected omega limit set $\omega\left(w_{0}\right)$. If $w^{*}$ denotes an arbitrary element of $\omega\left(w_{0}\right)$, then we have seen that the first component of $w^{*}$ is the zero function. Thus, we may assume that $w^{*}$ has the form

$$
w^{*}=\left(0, \ell^{*}(\cdot, \cdot)\right)
$$

for some $\ell^{*}(\cdot, \cdot) \in C(\bar{\Omega} \times[0, \tau+\sigma])$. Moreover, there exists an increasing sequence $\left\{t_{n}\right\}$ converging to infinity such that

$$
\lim _{t_{n} \rightarrow \infty} \ell\left(x, t_{n}, a\right)=\ell^{*}(x, a)
$$

uniformly in $\bar{\Omega} \times[0, \tau+\sigma]$. 
It is clear that if $n$ is sufficiently large, we can write

$$
t_{n}=m(n) T^{*}+T^{*}+t_{n}^{*}
$$

where $m(n) \in Z^{+}$and the residual $t_{n}^{*} \in\left[0, T^{*}\right]$. We can select a subsequence $t_{n^{\prime}}^{*}$ of residuals so that

$$
\lim _{n^{\prime} \rightarrow \infty} t_{n^{\prime}}^{*}=t^{*} \in\left[0, T^{*}\right]
$$

We relabel the convergent sequence of residuals $\left\{t_{n}^{*}\right\}$ and hence

$$
\lim _{n \rightarrow \infty} t_{n}^{*}=t^{*} \in\left[0, T^{*}\right]
$$

We observe that

$$
\ell\left(x, t_{n}, a\right)=\ell\left(x,(m(n)+1) T^{*}+t_{n}^{*}, a\right)
$$

and hence

$$
\ell^{*}(x, a)=\lim _{n \rightarrow \infty} \ell\left(x,(m(n)+1) T^{*}+t_{n}^{*}, a\right)
$$

By the representation formula (2.15), we have, for $n$ sufficiently large,

$$
\ell\left(x, t_{n}, a\right)=\left[V\left(t_{n}, t_{n}-a\right)\left(r\left(t_{n}-a\right) u\left(\cdot, t_{n}-a\right) v\left(\cdot, t_{n}-a\right)+h\left(\cdot, t_{n}-a\right)\right)\right](x) .
$$

From (2.6), we observe that

$$
\lim _{n \rightarrow \infty} V\left(t_{n}, t_{n}-a\right)=V\left(T^{*}+t^{*}, T^{*}+t^{*}-a\right)
$$

Also,

$$
\lim _{n \rightarrow \infty} r\left(t_{n}-a\right) u\left(\cdot, t_{n}-a\right) v\left(\cdot, t_{n}-a\right)=0
$$

uniformly on $\bar{\Omega} \times[0, \tau+\sigma]$, and

$$
\lim _{n \rightarrow \infty} h\left(\cdot, t_{n}-a\right)=h\left(\cdot, T^{*}+t^{*}-a\right) .
$$

Therefore, using (6.8), we have

$$
\begin{aligned}
\lim _{n \rightarrow \infty} \ell\left(x,(m(n)+1) T^{*}+t_{n}^{*}, a\right) & =\lim _{n \rightarrow \infty} \ell\left(x, t_{n}, a\right) \\
& =\left[V\left(T^{*}+t^{*}, T^{*}+t^{*}-a\right) h\left(\cdot, T^{*}+t^{*}-a\right)\right](x) .
\end{aligned}
$$



form

Hence, we have shown that any element belonging to the $\omega$-limit set $\omega\left(w_{0}\right)$ is of the

$$
w^{*}=\left(0, \ell^{*}(x, a)\right)
$$

and

$$
\begin{aligned}
\ell^{*}(x, a) & =\lim _{n \rightarrow \infty} \ell\left(x, t_{n}, a\right) \\
& =\left[V\left(T^{*}+t^{*}, T^{*}+t^{*}-a\right) h\left(\cdot, T^{*}+t^{*}-a\right)\right](x) .
\end{aligned}
$$

We observe that

$$
\left(0,\left[V\left(T^{*}+t^{*}, T^{*}+t^{*}-a\right) h\left(\cdot, T^{*}+t^{*}-a\right)\right](x)\right) \in \theta^{*}(x, a),
$$

and we have demonstrated that if $w(t)=\{u(\cdot, t), \ell(\cdot, t, \cdot)\}$ is a solution pair to (2.3a)-(2.3f), then

$$
\lim _{n \rightarrow \infty} \operatorname{dis}\left(w\left(t_{n}\right), \theta^{*}(\cdot, \cdot)\right)=0
$$

We remark that the pair

$$
\left(0,\left[V\left(T^{*}+t^{*}, T^{*}+t^{*}-a\right) h\left(\cdot, T^{*}+t^{*}-a\right)\right](x)\right)
$$

is periodic in $t$ with period $T^{*}$ and is a solution to the problem:

$$
\begin{aligned}
\frac{\partial u}{\partial t}(x, t) & =d_{1} \Delta u(x, t)-r(t) u(x, t) v(x, t), \quad x \in \Omega, \quad t>0, \\
\frac{\partial \ell}{\partial t}(x, t, a)+\frac{\partial \ell}{\partial a}(x, t, a) & =d_{2} \Delta \ell(x, t, a)-\lambda(t) \ell(x, t, a), x \in \Omega, t>0, a \in[0, \tau+\sigma]
\end{aligned}
$$

with

$$
\begin{aligned}
u(x, 0) & =0, \quad x \in \bar{\Omega} \\
\ell(x, 0, a) & =\left[V\left(T^{*}, T^{*}-a\right) h\left(\cdot, T^{*}-a\right)\right](x), x \in \bar{\Omega}, a \in[0, \tau+\sigma] \\
\ell(x, t, 0) & =h(x, t), \quad x \in \bar{\Omega}, \quad t>0
\end{aligned}
$$

and

$$
\frac{\partial u}{\partial n}=0, \quad \frac{\partial \ell}{\partial n}=0, \quad x \in \partial \Omega, \quad t>0, \quad a \in[0, \tau+\sigma] .
$$

Therefore, in case (b), we have characterized the attractor of bounded solutions to the initial-value problem (2.3a)-(2.3f). 
In conclusion, we have shown that under the assumption of periodicity in contact and mortality rates, and the addition of periodic source terms for both the susceptible and the infected populations, there exists a periodic solution of the SEIR model described by (2.3a)-(2.3f) (and the given hypothesis (i)-(vii)). We have also considered the cases where one or both source terms are zero, and further described the periodic solution in these cases.

We remark that, instead of the "mass action" term $r(t) u(x, t) v(x, t)$ of $(2.3 \mathrm{a})$ and (2.3f), a more general function $f(t, u, v)$ can be used to model the contact between susceptibles and infectives. We require $f$ to be nonnegative, polynomially bounded, $T^{*}$ periodic, and

$$
f(t, u, v) \geq r(t) u v
$$

on $\Omega \times\left[0, T^{*}\right]$. Then, using results from [14], we can show that all of our results obtained above are still valid. This more general approach will be the subject of future work. We also shall continue to investigate how other time-dependent distinguished solutions govern the dynamics of diffusive, age-dependent epidemic models. Of particular interest will be the assessment of the role played by almost periodic solutions.

\section{References}

1. W. E. Fitzgibbon, Semilinear functional differential equations in Banach space, J. Diff. Eqns. 29 (1978), 1-14.

2. W. E. Fitzgibbon, M. Langlais, M. E. Parrott, and G. F. Webb, A diffusive system with agedependence modeling HIV, J. Nonlinear Anal. TMA 25 (1995), 399-414.

3. W. E. Fitzgibbon, C. B. Martin, and J. J. Morgan, A diffusive epidemic model with criss-cross dynamics, J. Math. Anal. Appl. 184 (1994), 399-414.

4. W. E. Fitzgibbon, M. E. Parrott, and G. F. Webb, Diffusive epidemic models with spatial and age-dependent heterogeneity, Discrete and Continuous Dyn. Sys. 1 (1995), 35-57.

5. _ Diffusion epidemic models with incubation and criss-cross dynamics, Math. Biosci. 128 (1995), 131-135.

6. $358-369$

7. A. Friedman Partial Differential Equations, Holt, Rinehart and Winston, New York, 1969.

8. D. Gilbarg and N. S. Trudinger, Elliptic Partial Differential Equations of Second Order, SpringerVerlag, Berlin, 1970.

9. J. A. Goldstein, Semigroups of Linear Operators and Applications, Oxford University Press, New York, 1985.

10. J. K. Hale, Asymptotic Behavior of Dissipative Systems, American Math. Soc., Providence, Rhode Island, 1988.

11. P. Hess, Periodic-Parabolic Boundary-Value Problems and Positivity, Longman Sci. and Technical, Hurlow, Essex, UK, 1991.

12. M. Kubo and M. Langlais, Periodic solutions for a population dynamics problem with agedependence and spatial structure, J. Math. Biol. 29 (1991), 363-378.

13. __ Periodic solutions for nonlinear population dynamics models with age-dependence and spatial structure, J. Diff. Eqns. 109 (1994), 274-294.

14. J. J. Morgan and S. L. Hollis, The existence of periodic solutions to reaction-diffusion systems with periodic data, SIAM J. Math. Anal. 26 (1995), 1225-1232.

15. A. Pazy, Semigroups of Linear Operators and Applications to Partial Differential Equations, Springer-Verlag, Berlin, 1983.

16. J. Smoller, Shock Waves and Reaction Diffusion Systems, Springer-Verlag, Berlin, 1982.

17. P. E. Sobolevski, On equations of parabolic type in a Banach space, Trudy Mos. Mat. Obsc. 10 (1961), 297-350; translation in Amer. Math. Soc. Translations, Ser. 2 (1962), 1-62. 
18. K. E. Swick, Periodic solutions of a nonlinear age-dependent population model of single species, SIAM J. Math. Anal. 11 (1980), 901-910.

19. G. F. Webb, An age-dependent epidemic model with spatial diffusion, Arch. Rat. Mech. Anal. 75 (1980), 91-102.

20. _ , Theory of Nonlinear Age-Dependent Population Dynamics, Marcel Dekker, New York, 1985.

Department of Mathematics, University of Houston, Houston, TX 77204

E-mail: fitz@math.uh.edu

Department of Mathematics, Texas A\&M University, College Station, TX 77843

E-mail: jmorgan@math.tamu.edu

Department of Mathematics, University of South Florida, TAMPa, FL 33620-5700

E-mail: parrott@math.usf.edu 